\title{
Meixner classes and the square of white noise
}

\section{Accardi ${ }^{1}$}

Centro V. Volterra

Dipartimento di Matematica

Università degli Studi di Roma "Tor Vergata" Roma, Italy

Email: accardi@volterra.mat.uniroma2.it

WEB page: http://volterra.mat.uniroma2.it

\footnotetext{
${ }^{1}$ Talk given at the: AMS special session Analysis on Infinite Dimensional Spaces (in honor of L. Gross) during the AMS-MAA Joint Mathematics Meetings in New Orleans, LA, January 10-13, 2001.
} 


\section{Indice}

1 Emergence of white noise from stochastic limit 4

2 The square of white noise $\quad 8$

3 Classical infinitely divisible processes built from the SWN 12

4 Emergence of the square of white noise in different contexts 14

$\begin{array}{llr}5 & \text { Bibliography } & 18\end{array}$ 


\begin{abstract}
The introduction of a new (multiplicative) renormalization procedure leads to a Lie algebra for the square of white noise which turns out to be a current algebra on the Lie algebra $\operatorname{sl}(2, \mathbb{R})$. All the representations of this algebra enjoying a certain irreducibility property are constructed. A one parameter class of classical processes is defined in terms of the generators. The vacuum distributions of these processes are identified with the three exceptional (i.e. non Gaussian or Poisson) classes in the Meixner classification. This class of distributions has been recently studied by several authors in connection with different problems arising in decision theory, mathematical finance, quantum field theory, classical probability, .... In the last section of the paper these developments will be quickly reviewed.
\end{abstract}




\section{Emergence of white noise from stochastic limit}

In this section the main steps which lead from the stochastic limit of quantum theory [AcLuVo02] to the white noise approach to stochastic calculus will be described and it will be explained why this development naturally leads to conceive the possibility of a calculus for higher powers of white noise (nonlinear stochastic calculus).

The following is a relatively simple result which plays a fundamental role in the stochastic limit of quantum theory. For its proof, as well as for the proof of all the statements in this section, we refer to [AcLuVo02]. The theory works equally well for Boson and Fermion fields, but in this paper we shall discuss only the Boson case.

Theorem 1 Let $a_{k}, a_{k^{\prime}}^{+}$be a Boson Fock field:

$$
\left[a_{k}, a_{k^{\prime}}^{+}\right]=\delta\left(k-k^{\prime}\right) \quad ; \quad a_{k} \Phi=0
$$

and let $\omega_{k}$ be a function and $\omega$ a real number. Then, in the sense of operator valued distributions on test functions satisfying

$$
\left.\int_{\mathbb{R}} d t\left|\int_{\mathbb{R}^{3}} d k\right| g(k)\right|^{2} e^{i t\left(\omega_{k}-\omega\right)} d k \mid<\infty
$$

one has

$$
\begin{gathered}
\lim _{\lambda \rightarrow 0} \frac{e^{i\left(t / \lambda^{2}\right) \omega_{k}}}{\lambda} a_{k}^{+}=b_{\omega}(t, k) \\
{\left[b(t, k), b^{+}\left(t^{\prime}, k^{\prime}\right)\right]=2 \pi \delta\left(t-t^{\prime}\right) \delta\left(\omega_{k}-\omega_{0}\right) \delta\left(k-k^{\prime}\right)}
\end{gathered}
$$

Definition 1 A quantum field $b(t, k)$ satisfying

$$
\left[b(t, k), b^{+}\left(t^{\prime}, k^{\prime}\right)\right]=\delta\left(t-t^{\prime}\right) G\left(k, k^{\prime}\right)
$$

$G\left(k, k^{\prime}\right)$ positive definite distribution on $\mathbb{R}^{d} \times \mathbb{R}^{d}$ is called a Boson white noise. If, in the representation space of the field, there exists a unit (vacuum) vector $\Phi$ such that

$$
b(t, k) \Phi_{0}=0
$$

then $b(t, k)$ is called a Boson Fock white noise. 
Notice that, defining

$$
b(t, k)+b^{+}(t, k)=: w(t, k)
$$

denoting $\mathcal{K}$ the completion of the Shwartz space $\mathcal{S}\left(\mathbb{R}^{d}\right)$ for the scalar product

$$
\langle\varphi, \psi\rangle:=\iint \bar{\varphi}(k) G\left(k, k^{\prime}\right) \psi\left(k^{\prime}\right) d k d k^{\prime}
$$

and defining

$$
w(t, f):=\int f(k) w(t, k) d k ; \quad f \in \mathcal{K}
$$

Then, with respect to the vacuum vector $\Phi$, i.e. in the sense of the expectation value

$$
\langle X\rangle:=\langle\Phi, X \Phi\rangle
$$

$w(t, f)$ is a $\mathcal{K}$-valued classical white noise, in the sense that

$$
\left[w(t, f), w\left(t^{\prime}, g\right)\right]=0 \quad ; \quad \forall t, t^{\prime}, f, g
$$

with covariance

$$
\left\langle w(t, f), w\left(t^{\prime}, g\right)\right\rangle=\delta\left(t-t^{\prime}\right)\langle f, g\rangle
$$

Let $\mathcal{H}_{R}$ denote the Fock space of the Boson field and let $\mathcal{H}_{S}$ be the state space of a system (say a lattice of atoms) interacting with the field with a dipole type interaction.

The dynamics of such a system is described, in interaction representation, by a Schrödinger equation of the form

$$
\partial_{t} U_{t}^{(\lambda)}=-i \lambda\left(D \otimes \int_{\mathbb{R}^{3}} d k g(k) a_{k}^{+} e^{i t\left(\omega_{k}-\omega_{0}\right)}+h . c .\right) U_{t}^{(\lambda)}
$$

where $\omega_{k} \geq 0$ is the energy density of the field, $D$ is an operator acting on the system space $\mathcal{H}_{S}, \lambda$ is a coupling constant and $\omega_{0}$ is a proper frequency of the system. For example, for a 2-level atom $\omega_{0}$ is the energy difference between the upper and the lower level. In general these proper frequencies are infinitely many, but we are discussing the simplest situation.

After the time rescaling $t \rightarrow t / \lambda^{2}$ the evolution equation becomes

$$
\partial_{t} U_{t / \lambda^{2}}^{(\lambda)}=-i\left(D \otimes \int_{\mathbb{R}^{3}} d k g(k) \frac{e^{i\left(t / \lambda^{2}\right)\left(\omega_{k}-\omega_{0}\right)}}{\lambda} a_{k}^{+}+\text {h.c. }\right) U_{t}
$$

At this point a naive application of Theorem (1) suggests the following natural conjecture: 
Theorem $2 A s \lambda \rightarrow 0$ the solution $U_{t / \lambda^{2}}^{(\lambda)}$ of equation (1) converges (in a sense to be specified) to the unitary solution $U_{t}$ of the white noise Hamiltonian (WNH) equation

$$
\partial_{t} U_{t}=-i H_{I}(t) U_{t}=-i\left(D \otimes b_{t}^{+}+D^{+} \otimes b_{t}\right) U_{t}
$$

where $b(t, k)$ is the white noise obtained in Theorem (1) and

$$
b_{t}^{+}:=\int d k g_{k} b^{+}(t, k)
$$

In particular, if $D=D^{*}$ then equation (2) becomes

$$
\partial_{t} U_{t}=\frac{d U}{d t}=-i\left(D \otimes w_{t}\right) U_{t}
$$

where $w_{t}:=b_{t}^{+}+b_{t}$ is a classical white noise.

Remark. A corollary of the above result is that white noise is not the derivative of Brownian motion in the naive sense that, by replacing an ordinary white noise differential equation by the corresponding integral equation, one gets a stochastic differential equation. In fact, if we consider the integral equation, associated to the Hamiltonian White Noise equation (3) one obtains

$$
U_{t}=1+(-i)\left(D \otimes \int_{0}^{t} w_{s} d s U_{s}\right)
$$

and, if it were true that

$$
w_{s} d s=d W_{s} \quad(W-B M)
$$

then (3) should be equivalent to

$$
U_{t}=1+(-i)\left(D \otimes \int_{0}^{t} d W_{s} U_{s}\right)
$$

in the sense of usual stochastic integrals. But (4) is equivalent to the SDE

$$
d U_{t}=-i\left(D \otimes d W_{t}\right) U_{t}
$$

Theorem 3 : (3) is not equivalent to (5). 
Proof. The solution of (5) cannot be unitary, unless $D$ is zero.

On the other hand, starting from [AcFrLu87], we know that

Theorem 4 As $\lambda \rightarrow 0$ the solution $U_{t / \lambda^{2}}^{(\lambda)}$ of equation (1)converges (in a sense to be specified) to the solution $U_{t}$ of the quantum stochastic differential equation

$$
d U_{t}=-i\left(D \otimes d B_{t}^{+}+D^{+} \otimes d B_{t}+\gamma_{-} D^{*} D d t\right) U_{t}
$$

From this we deduce that the two equations (2) and (4) must be equivalent and that there should be a simple rule allowing to pass from one to the other. The correctness of this conjecture, first proved in [AcLuVo93], is shown by the following:

Theorem 5 The causally normally ordered form of the WNH equation

$$
\partial_{t} U_{t}=-i\left(D \otimes b_{t}^{+}(g)+h . c .\right) U_{t}
$$

is the $S D E$

$$
\frac{d U}{d t}=-i\left(D \otimes b_{t}^{+}\right) U_{t}-i D U_{t} b_{t}+\gamma_{-} D^{+} D U_{t}
$$

where

$$
\gamma_{-}:=\int_{-\infty}^{0} d t \int d k|g(k)|^{2} e^{i t(\omega(k)-\omega)}
$$

For the notion of causal normal order we refer to [AcLuVo02].

To complete the inclusion:

Stochastic Differential Equations (Classical or Quantum) "ᄃ" Hamiltonian white noise equation

a white noise formulation of the Ito formula had to be developed. It turns out [AcLuVo95b] that this formulation can be synthetized in the formal identity

$$
\delta(0) d t=1
$$

in the sense that one can give a rigorous meaning to this identity by means of several different limiting procedures (which are the analytical counterpart of the many different notions of stochastic integral) and that the stochastic limit is one of these possibilities. 


\section{The square of white noise}

The fact that the white noise itself does not enter explicitly in the white noise Ito formula (8) suggested the possibility of a naive application of this formula to higher powers of white noise thus obtaining a nonlinear extension of the ususal Ito calculus. In fact, already starting from $2-d$ order WNE we are beyond usual stochastic calculus.

This program was initiated in [AcLuVo95b] and carried out in several directions but it soon turned out that a too naive application of this formula lead to some unpleasant features, such as the non associativity of the non linear Ito tables.

The idea to replace additive renormalization by multiplicative renormalization [AcLuVo99] [AcLuVo00] allowed to construct the Fock representation for the the renormalized square of white noise (SWN in the following). The essence of the new idea can be summarized as follows:

Since the usual Ito multiplication tables are consequences of the commutation relations, rather than renormalizing directly the Ito table one might try to renormalize the commutation relations themselves.

This idea was substantiated, in the following Lemma, by making use of the known formula of distribution theory

$$
\delta(t)^{2}=c \delta(t)
$$

where the constant $c \in \mathbb{C}$ is arbitrary (for a proof of this formula and bibliographical references, cf. [AcLuVo99]).

Lemma 1 Let $\mathcal{P}\left(b, b^{+}\right)$denote the free associative unital complex $*$-algebra generated by the symbols $b_{t}, b_{t}^{+}$with $t \in \mathbb{R}$ and commutation relations

$$
\left[b_{t}, b_{\tau}^{+}\right]=\delta(t-\tau)
$$

and let us define the square of the $\delta$-function by formula (9) with an arbitrary constant $c$. Then the following commutation relations hold:

$$
\begin{gathered}
{\left[b_{t}^{2}, b_{t^{\prime}}^{+2}\right]=2 \delta\left(t-t^{\prime}\right) c+4 b_{t}^{+} b_{t} \delta\left(t-t^{\prime}\right)} \\
{\left[b_{t}^{+} b_{t}, b_{t^{\prime}}^{+2}\right]=2 \delta\left(t-t^{\prime}\right) b_{t^{\prime}}^{+2}}
\end{gathered}
$$

In particular $b_{t}^{+2}, b_{t}^{2}, b_{t}^{+} b_{t}$ have a closed Lie algebra. 


\section{Proof.}

$$
\begin{gathered}
{\left[b_{t}^{2}, b_{t^{\prime}}^{+2}\right]=\left[b_{t}^{2}, b_{t^{\prime}}^{+}\right] b_{t^{\prime}}^{+}+b_{t^{\prime}}^{+}\left[b_{t}^{2}, b_{t^{\prime}}^{+}\right]=} \\
=b_{t}\left[b_{t}, b_{t^{\prime}}^{+}\right] b_{t^{\prime}}^{+}+\left[b_{t}, b_{t^{\prime}}^{+}\right] b_{t} b_{t^{\prime}}^{+}+b_{t^{\prime}}^{+} b_{t}\left[b_{t}, b_{t^{\prime}}^{+}\right]+b_{t^{\prime}}^{+}\left[b_{t}, b_{t^{\prime}}^{+}\right] b_{t}= \\
=\delta\left(t-t^{\prime}\right) b_{t} b_{t^{\prime}}^{+}+\delta\left(t-t^{\prime}\right) b_{t} b_{t^{\prime}}^{+}+b_{t^{\prime}}^{+} b_{t} \delta\left(t-t^{\prime}\right)+b_{t^{\prime}}^{+} \delta\left(t-t^{\prime}\right) b_{t}= \\
=2 \delta\left(t-t^{\prime}\right) b_{t} b_{t}^{+}+2 \delta\left(t-t^{\prime}\right) b_{t}^{+} b_{t}=2 \delta\left(t-t^{\prime}\right)^{2}+4 \delta\left(t-t^{\prime}\right) b_{t^{\prime}}^{+} b_{t}
\end{gathered}
$$

Using the relation (9) we find (11). Similarly

$$
\begin{gathered}
b_{t}^{+} b_{t} b_{t^{\prime}}^{+2}=b_{t}^{+}\left[b_{t}, b_{t^{\prime}}^{+2}\right]+b_{t}^{+} b_{t^{\prime}}^{+2} b_{t}=b_{t}^{+} \delta\left(t-t^{\prime}\right) b_{t^{\prime}}^{+}+b_{t}^{+} b_{t^{\prime}}^{+} \delta\left(t-t^{\prime}\right)+b_{t^{\prime}}^{+2} b_{t}^{+} b_{t}= \\
=2 \delta\left(t-t^{\prime}\right) b_{t}^{+} b_{t^{\prime}}^{+}+b_{t^{\prime}}^{+2} b_{t}^{+} b_{t}
\end{gathered}
$$

which is (12).

Lemma 3 suggests to introduce a Lie algebra with generators

$$
B_{t}^{+} \quad, \quad B_{t} \quad, \quad N_{t}
$$

and a central element $E$ (which we omit from notations) with relations:

$$
\begin{gathered}
{\left[B_{t}, B_{\tau}^{+}\right]=2 c \delta(t-\tau)+4 \delta(t-\tau) N_{\tau}} \\
{\left[N_{t}, B_{\tau}^{+}\right]=2 \delta(t-\tau) B_{\tau}^{+}} \\
{\left[N_{t}, B_{\tau}\right]=-2 \delta(t-\tau) B_{\tau}} \\
{\left[N_{t}, N_{\tau}\right]=\left[B_{t}, B_{\tau}\right]=\left[B_{t}^{+}, B_{\tau}^{+}\right]=0}
\end{gathered}
$$

and to construct a representation of this Lie algebra with a cyclic vacuum $\Phi$, i.e. a unit vector satisfying

$$
B_{t} \Phi=0
$$

and such that the quadratic number vectors

$$
B_{t_{n}}^{+} \ldots B_{t_{1}}^{+} \Phi
$$

are total in the representation space. The above Lie algebra is called the SWN algebra and a representation with the above properties, a Fock representation. Intuitively

$$
B_{\tau}^{+}:=b_{t}^{+2}=b_{t}^{+} b_{t}^{+} \quad, \quad B_{t}=b_{t}^{2}=b_{t} b_{t} \quad, \quad N_{t}=b_{t}^{+} b_{t}
$$

and the central element $E$ is the identity (of the universal enveloping algebra). 
In [AcLuVo99] it was proved that a Fock representation of the SWN algebra exists if and only if the constant $c$, in formula (9), is strictly positive.

This opened the way to a multiplicity of developments starting from the paper of P. Śniady [Śnia99] where the $q$-deformation of the SWN was discussed, the free $(q=0)$ Fock representation explicitly constructed and it was proved that there exist no natural Fock representation for both the first and the second order white noise (in other words: the renormalization procedure has indeed a strong effect on the relations between the first and second order white noises).

Simultaneously L. Accardi and M. Skeide [AcSk99a], [AcSk99b] introduced the exponential vectors for the SWN and used them to prove that the Fock space of the SWN was isomorphic to the Fock space of the finite difference algebra, previously constructed, on the basis of completely different motivations, by A. Boukas and P. Feinsilver [Bou88], [Bou91], [Fei87], [FeSch93].

This apparently surprising connection was explained in the paper by L. Accardi, U. Franz, M. Skeide [AcFrSk00] where it is shown that the Fock representation of the SWN realizes a factorizable representation of a current algebra of the real Lie algebra $s l(2, \mathbb{R})$ over $\mathbb{R}$ (in fact all the main results of this paper are applicable to a more general, multidimensional index set) in which the three generators $B^{ \pm}, M$ correspond respectively to the formal expressions $b^{+2} b^{2}, b^{+} b$ where $b^{ \pm}$is a usual quantum white noise.

In fact, after introducing test functions in the usual way

$$
b_{\varphi}^{+} "=" \int d t \varphi(t) b_{t}^{2} \quad ; \quad b_{\varphi}=\left(b_{\varphi}^{+}\right)^{+} ; \quad n_{\varphi} "=" \int d t \varphi(t) b_{t}^{+} b_{t}
$$

and appropriately fitting the parameters, the commutation relations become:

$$
\begin{gathered}
{\left[b_{\varphi}, b_{\psi}^{+}\right]=\gamma\langle\varphi, \psi\rangle+n_{\bar{\varphi} \psi}} \\
{\left[n_{\varphi}, b_{\psi}\right]=-2 b_{\bar{\varphi} \psi}} \\
{\left[n_{\varphi}, b_{\psi}^{+}\right]=2 b_{\varphi \psi}^{+}} \\
\left(b_{\varphi}^{+}\right)^{+}=b_{\varphi} \quad ; \quad n_{\varphi}^{+}=n_{\bar{\varphi}}
\end{gathered}
$$

Comparing this with the relations among the three generators $B^{-}, B^{+}, M$ of the Lie algebra $\operatorname{sl}(2, \mathbb{R})$ :

$$
\left[B^{-}, B^{+}\right]=M \quad ; \quad\left[M, B^{ \pm}\right]= \pm 2 B^{ \pm}
$$


we see that the SWN algebra is isomorphic to (a central extension of) the Lie algebra $\operatorname{sl}(2, \mathbb{R})$.

Moreover the commutation relations (19), (20), (21), (22) clearly imply that operators, corresponding to test functions with disjoint support commute. This, together with (17), implies that they are independent random variables with respect to the vacuum.

The intuitive interpretation of this processes as naturally associated to the SWN can give some insight on their distributions. Let us illustrate this with the example of the renormalized square of the classical white noise. The classical $\mathrm{WN}$ is the independent increment process

$$
w_{t}=b_{t}^{+}+b_{t}=w_{[0, t]}=\Sigma_{j} w_{\left[t_{j+1}-t_{j}\right]}
$$

Computing formally its square we find

$$
w_{t}^{2}=\left(b_{t}^{+}+b_{t}\right)^{2}=b_{t}^{+2}+b_{t}^{2}+b_{t}^{+} b_{t}+b_{t} b_{t}^{+}=b_{t}^{+2}+b_{t}^{2}+2 b_{t}^{+} b_{t}+\delta(0)
$$

thus, after additive renormalization (i.e. subtraction of the infinity $\delta(0)$ ) we find:

$$
: w_{t}^{2}:=b_{t}^{+2}+b_{t}^{2}+2 b_{t}^{+} b_{t}
$$

which, by the above remark is also an independent increment, i.e. a sum of squares of independent Gaussians:

$$
: w_{t}^{2}:=: w_{[0, t]}^{2}:=\Sigma: w_{\left[t_{j}-t_{j}\right]}^{2}:
$$

therefore, if the above formal manipulations lead to the correct intuition the vacuum distribution of : $w_{t}^{2}$ : should be a $\chi^{2}$-distribution. This means that, after integration with a test function $\varphi$

$$
\int \varphi(t): w_{t}^{2}: d t=b_{\varphi}^{+}+b_{\varphi}+n_{\varphi}=: w_{\varphi}^{2}
$$

we have to check, using the commutation relations, that we obtain a classical (commuting) process and that its distribution is a $\chi^{2}$. We will see that this is indeed the case.

We have seen that to any representation of the SWN algebra it is associated an independent increment processes on $\mathbb{R}$. According to the general theory of independent increment processes on $*$-bialgebras [AcSchuvW88], [Sch93], such processes are classified by a representation $\rho$ of the Lie algebra $\operatorname{sl}(2, \mathbb{R})$ and a $\rho$-cocycle $\eta$. 
Using the theory of unitary representations of $S L(2, \mathbb{R})$, in $[\mathrm{AcFrSk} 00]$ all such pairs, corresponding to an irreducible $\rho$ were classified.

In [AcAmFr02] an explicit classification was obtained of the SWN analogue of the Bogolyubov automorphisms. This allowed to define the notion of free evolution for this algebra and to study the corresponding KMS states which should correspond to the equilibrium representations of the SWN algebra. A different construction of the (Fock) SWN process has recently been obtained by [Priv02].

\section{Classical infinitely divisible processes built from the SWN}

The last part of [AcFrSk00] is devoted to the study of those classical infinitely divisible processes built from the SWN in a similar way as the Wiener and the Poisson processes are built from the usual WN. These have the form

$$
X_{\beta}(t):=b_{t}^{+2}+b_{t}^{2}+\beta b_{t}^{+} b_{t}=B_{t}^{+}+B_{t}+\beta N_{t}
$$

where $\beta$ is a real number (because we want formally self-adjoint processes). Without the power 2 , for $\beta=0$ one would find the Wiener (or field) process and, for $\beta \neq 0$, the Poisson process of intensity $\beta$.

With the power 2 , the only privileged parameters are $\beta= \pm 2$ : the value +2 corresponding to the renormalized square of the position (classical) white noise, i.e.

$$
\begin{gathered}
\left|b_{t}^{+}+b_{t}\right|^{2}=b_{t}^{+2}+b_{t}^{2}+b_{t}^{+} b_{t}+b_{t} b_{t}^{+}+ \\
=b_{t}^{+2}+b_{t}^{2}+2 b_{t}^{+} b_{t}+\delta(0) \equiv b_{t}^{+2}+b_{t}^{2}+2 b_{t}^{+} b_{t}
\end{gathered}
$$

and the value -1 to the renormalized square of the momentum white noise, i.e. $\left(b_{t}^{+}-b_{t}\right) / i$. In $[\mathrm{AcFrSk} 00]$ it was proved that the vacuum distribution of both processes is the Gamma-distribution

$$
\mu(\mathrm{d} x)=\frac{|x|^{m_{0}-1}}{\Gamma\left(m_{0}\right)} e^{-\beta x} \mathbf{1}_{\beta \mathbb{R}_{+}}
$$

whose parameter $m_{0}>0$ is uniquely determined by the choice of the unitary representation of $S L(2, \mathbb{R})$ corresponding to the representation of the SWN algebra. Since the Gamma-distributions are precisely the distributions of the $\chi^{2}$-random variables, this result confirms the naive intuition about the 
distribution of the renormalized square of white noise which was discussed in the previous section. Notice that the above expression of the gamma function corresponds to a reparametrisation in which these two processes correspond to the choice $|\beta|=1$ rather than $|\beta|=2$.

For any value of $\beta$ the process $X=X_{\beta}$ (everything depends on $\beta$, but we omit this dependence from our notation) is classical and, denoting $\mu$ its vacuum distribution, and $P_{n}(x)$ the associated sequence of orthogonal polynomials, with initial condition $P_{-1}=0, P_{1}=1$, using the commutation relations one can verify that the Jacobi recurrence relation satisfied by the $P_{n}(x)$ is

$$
(n+1) P_{n+1}+\left(2 \beta n+\beta m_{0}-x\right) P_{n}+\left(n+m_{0}-1\right) P_{n-1}=0
$$

For $|\beta|=1$ this leads, up to appropriate rescalings, to

$$
P_{n}(x)=(-\beta)^{n} L_{n}^{\left(m_{0}-1\right)}(\beta x)
$$

where $L_{n}^{(\alpha)}$ are the Laguerre polynomials which are orthogonal for the gamma distribution.

For $|\beta|<1$

$$
P_{n}(x)=P_{n}^{\left(m_{0} / 2\right)}\left(\frac{x}{2 \sqrt{1-\beta^{2}}} ;-\arccos \beta\right)
$$

where $P_{n}^{\left(m_{0} / 2\right)}$ are the Meixner-Pollaczek polynomials corresponding to the measure

$$
\mu(\mathrm{d} x)=C \exp \left(-\frac{(2 \arccos \beta+\pi) x}{2 \sqrt{1-\beta^{2}}}\right)\left|\Gamma\left(\frac{m_{0}}{2}+\frac{i x}{2 \sqrt{1-\beta^{2}}}\right)\right|^{2}
$$

where $C$ is a normalization constant. For $m_{0}$ integer there are explicit formulae for the densities because it is known that (cf. [Grig01])

$$
\begin{gathered}
|\Gamma(n+i x)|^{2}=\frac{\pi x\left(1+x^{2}\right) \ldots\left((n-1)^{2}+x^{2}\right)}{\sinh (\pi x)} \quad, \quad n=1,2, \ldots, \quad x \in \mathbb{R} \\
\left|\Gamma\left(\frac{1}{2}+i x\right)\right|^{2}=\frac{\pi}{\cosh (\pi x)} \quad, \quad x \in \mathbb{R}
\end{gathered}
$$




$$
\left|\Gamma\left(n+\frac{1}{2}+i x\right)\right|^{2}=\frac{\pi\left(\frac{1}{4}+x^{2}\right) \ldots\left((n-1) n+\frac{1}{4}+x^{2}\right)}{\cosh (\pi x)} \quad, \quad n=1,2, \ldots ; x \in \mathbb{R}
$$

Finally, for $|\beta|>1$

$$
P_{n}(x)=\left\{\begin{array}{llc}
(-1)^{n} \prod_{k=1}^{n} \frac{n+m_{0}-1}{n} M_{n}\left(\frac{x}{c-1 / c}-\frac{m_{0}}{2} ; m_{0} ; c^{2}\right) & \text { if } & \beta>0 \\
\prod_{k=1}^{n} \frac{n+m_{0}-1}{n} M_{n}\left(-\frac{x}{c-1 / c}+\frac{m_{0}}{2} ; m_{0} ; c^{2}\right) & \text { if } & \beta>0
\end{array}\right.
$$

where the $M_{n}$ are the Meixner polynomials and

$$
c= \begin{cases}\beta-\sqrt{\beta^{2}-1} & \text { if } \quad \beta>+1 \\ -\beta-\sqrt{\beta^{2}-1} & \text { if } \quad \beta<-1\end{cases}
$$

The associated probability measure is the negative binomial (Pascal) distribution

$$
\mu=C \sum_{n=0}^{\infty} \frac{c^{2 n}\left(m_{0}\right)_{n}}{n !} \delta_{\operatorname{sgn} \beta\left((c-1 / c)\left(n+m_{0} / 2\right)\right)}
$$

where $\left(m_{0}\right)_{n}$ denotes the Pochammer symbol,

$\left(m_{0}\right)_{n}=m_{0}\left(m_{0}+1\right) \cdots\left(m_{0}+n-1\right)$ and

$$
C^{-1}=\sum_{n=0}^{\infty} \frac{c^{2 n}\left(m_{0}\right)_{n}}{n !}=\left(1-c^{2}\right)^{-m_{0}}
$$

\section{Emergence of the square of white noise in different contexts}

In this section we prove that the classical processes (23), naturally associated to the SWN exactly coincide with the three exceptional (i.e. neither Gaussian nor Poisson) Meixner classes. Following [Lytv02a] let us briefly recall the main facts about these classes.

In 1934 Meixner considered the following problem [Meix34]: Find all sequences of polynomials $P^{(n)}(x)(n \in \mathbb{N})$, in one real variable $x$ with the following properties:

(i) the leading coefficient of each $P^{(n)}(x)$ is 1 
(ii) for each $n \in \mathbb{N}, P^{(n)}(x)$ is the $n$-th orthogonal polynomial with respect to some probability measure $\mu$ on $\mathbb{R}$.

(iii) there exist functions $f(z)$ and $\Psi(z)$ such that

$$
G(x, z):=\exp (x \Psi(z)) f(z)=\sum_{n=0}^{\infty} \frac{P^{(n)}(x)}{n !} z^{n}
$$

The sequence $\left(P^{(n)}\right)$ of orthogonal polynomials, with respect to any probability measure $\mu$ on $\mathbb{R}$, is characterized by the Jacobi recurrence formula (Favard theorem)

$$
\begin{gathered}
x P^{(n)}(x)=P^{(n+1)}(x)+a_{n} P^{(n)}(x)+b_{n} P^{(n-1)}(x) \quad, \quad n \in \mathbb{N} \\
P^{(-1)}(x):=0 \quad, \quad P^{(0)}(x):=1
\end{gathered}
$$

where $a_{n}$ are real number and $b_{n}$ are positive numbers which define a unique infinite (symmetric) Jacobi matrix with the $a_{n}$ on the main diagonal and the $\sqrt{b_{n}}$ on the upper and lower diagonals.

Meixner proved that (34) implies that

$$
\Psi^{-1}(D) P^{(n)}(x)=n P^{(n-1)}(x), \quad n \in \mathbb{N}
$$

where $\Psi^{-1}$ is the inverse function of $\Psi$ and $D:=\frac{d}{d x}$. and using this he showed that the solution of this problem is completely determined by two parameters $\lambda$ and $k$ satisfying the equations

$$
\begin{gathered}
k=\frac{b_{n}}{n}-\frac{b_{n-1}}{n-1} \quad, \quad n \geq 2 \\
a_{n}=\lambda n
\end{gathered}
$$

According to the values of the parameters $k, \lambda$ the solutions of these equations, hence the corresponding measures, can be classified in 5 classes: the Meixner classes.

The first two classes correspond to the case $k=0$, so that $b_{n}=n$.

(I) $\lambda=0$, so that $a_{n}=0$

In this case (35) becomes the recurrence relation of the Hermite polynomials and $\mu$ is the standard Gaussian distribution on $\mathbb{R}$.

(II) $\lambda \neq 0$ 
In this case (35) becomes the recurrence relation of the Charlier polynomials and $\mu$ is the centered Poisson distribution on $\mathbb{R}$ with parameter $\lambda$.

The remaining 3 classes correspond to the case $k \neq 0$. In this case

$$
b_{n}=k n+\frac{n}{n-1} b_{n-1}=k n+\frac{n}{n-1}\left(k(n-1)+\frac{n-1}{n-2} b_{n-2}=\ldots=k n^{2}\right.
$$

Following [Lytv02a] we set $k=1$ and introducing two complex numbers $\alpha$ and $\beta$ through the equation

$$
1+\lambda z+z^{2}=(1-\alpha z)(1-\beta z) .
$$

we distinguish the three following cases:

(III) $|\lambda|=2$, so that $\alpha=\beta= \pm 1$ (these two cases correspond to the renormalized square of classical white noise which was discussed above).

In this case the $P^{(n)}(x)$ are the Laguerre polynomials and $\mu$ is a centered gamma distribution which is a compound Poisson measure. These measures do not possess the chaotic decomposition property but, as shown in $[\mathrm{Nu}-$ Sch00] (see also the recent book [Schou00]), they enjoy a generalization of this property obtained by adding to the original process $\left(X_{t}\right)$ its associated power jump processes

$$
X_{t}^{(i)}:=\sum_{0<s \leq t}\left(\Delta X_{s}\right)^{(i)} \quad, \quad i \geq 2
$$

The gamma, Pascal, and Meixner processes served as main examples of generalized chaotic representation for square-integrable random variables in terms of the orthogonalized Teugels martingales related to the process.

The one-dimensional polynomials of Meixner's type were used in this paper in order to carry out the orthogonalization procedure of the Teugels martingales (which, in turn, are the centered power jump processes related to the original process).

The connection between the gamma processes and the current representations of $S L(2, \mathbb{R})$ was noticed in [TsVeYo01] independently of [AcFrSk00], where this connection was established for all the Meixner classes.

(IV) $|\lambda|>2$, so that $\alpha \neq \beta$.

In this case the $P^{(n)}(x)$ are the Meixner polynomials (of the first kind), which are orthogonal with respect to a centered Pascal (negative binomial) distribution i.e., up to reparametrization, a distribution of the form (33). 
The Lévy processes, corresponding to the Pascal measures were introduced in [BruRo91] in the context of optimal selection strategies based on relative ranks, when the total number of options is unknown.

(V) $|\lambda|<2$, so that $\alpha \neq \beta$, both complex conjugate.

In this case the $P^{(n)}(x)$ are the Meixner polynomials of the second kind, (or Meixner-Pollaczek polynomials). These are orthogonal with respect to a measure $\mu$ obtained by centering a probability measure of the form $C \exp (a x)|\Gamma(1+i m x)|^{2} d x$, where $a \in \mathbb{R}, m>0$, and $C$ is the normalizing constant. These are called Meixner measures in [Lytv02a] and they are a sub-class of Grigelionis' generalized $z$-distributions [Grig01]. In this paper the term Meixner distribution is used for the class of probability measures on $\mathbb{R}$ whose characteristic function (Fourier transform of the probability density) has the form

$$
\hat{f}(z)=\left(\frac{\cos (\beta / 2)}{\cosh ((\alpha z-i \beta) / 2)}\right)^{2 \delta}
$$

with $z \in \mathbb{R},-\pi<\beta<\pi, \delta>0, \mu \in \mathbb{R}$. This class was introduced in [SchTeu98] where it was proved that the measures in it correspond to Levy processes and their connection with the Meixner-Pollaczek polynomials was established. We refer to [Grig01], [Grig00], [Grig99] for several interesting properties of these distributions and explicit formulae related to them. In particular, in [Grig99], the Meixner process was proposed as a model for risky assets and an analogue of the Black and Sholes formula was established for them.

In all the above cases, the generating function $G(x, z)$ defined in (34) can be represented as

$$
G(x, z)=\exp (x \Psi(z)) / \ell_{\mu}(\Psi(z))
$$

where

$$
\ell_{\mu}(z):=\int_{\mathbb{R}} e^{z x} \mu(d x)
$$

is the extension of the Laplace transform of the measure $\mu$ defined in a neighborhood of zero in $\mathbb{C}$.

The infinite dimensional and multidimensional analogues of orthogonal polynomials associated to a given measure have been widely studied both in the Gaussian ([BeKo94], [HiKuPoStr93], [Kuo96]) and in the Poisson case ([Chiha78], [KoKuOl00]). 
The programme to extend this analysis to more general probability measures was developed by Berezansky, who introduced in this connection the notion of Jacobi field of operators, and his school ([BeLiLy95], [Lytv95], [Berez98], [Berez97]).

An infinite-dimensional analogue of the Laguerre polynomials and the corresponding Jacobi fields, corresponding to the gamma case, i.e. to the class (III) in Meixner's classification, was studied in [KoLy00], see also [KoSiSt97].

In conclusion it should be added that the SWN was introduced as an example of interacting Fock space and in the attempt to extend to infinite dimensions the canonical connection between orthogonal polynomials and interacting Fock spaces established, in the 1-dimensional case, in [AcBo98].

Now this connection has been established in the paper [AcKuSt02] and it will be discussed elsewhere. Also in this case there are interesting connections with previous papers by Yu. Berezansky and by E. Lytvinov.

\section{Bibliography}

[AcKuSt02] L. Accardi, H.-H. Kuo:

Orthogonal polynomials and interacting Fock space, in preparation

[AcLuVo02] Accardi L., Y.G. Lu, I. Volovich:

Quantum Theory and its Stochastic Limit.

Springer Verlag (2002) Japanese translation, Tokyo-Springer (2002)

[AcAmFr02] Luigi Accardi, Grigori Amosov, Uwe Franz:

KMS states of the square of white noise algebra,

Preprint Volterra (2002)

[AcFrSk00] L. Accardi, U. Franz, M. Skeide:

Renormalized squares of white noise and other non-gaussian noises as Lévy processes on real Lie algebras,

Comm. Math. Phys. (2002) Preprint Volterra, N. 423 (2000)

[AcSk99b] Luigi Accardi, Michael Skeide: 
On the Relation of the Square of White Noise and the Finite Difference Algebra,

IDA-QP 3 (2000) 185-189 Volterra Preprint N. 386 (1999)

[AcSk99a] L. Accardi and M. Skeide:

Hilbert module realization of the Square of White Noise and the Finite Difference algebra,

Math. Notes (1999)

Volterra Preprint N. 384 (1999)

[AcLuVo00] Accardi, L., Lu, Y.G., Volovich, I.V.:

A white noise approach to stochastic calculus,

Acta Applicandae Mathematicae 63 (2000) 3-25

[AcLuVo99] Accardi, L., Lu, Y.G., Volovich, I.V.:

A white noise approach to classical and quantum stochastic calculus, Volterra Preprint N.375 July (1999)

World Scientific (2001) to appear

[AcBo98] Luigi Accardi, Marek Bożejko:

Interacting Fock spaces and Gaussianization of probability measures

Infinite dimensional analysis, quantum probability and related topics, 1, N. 4 (1998) 663-670 Volterra Preprint (1997) N. 321

[AcLuVo97b] Accardi L., Lu Y.G., I. Volovich

The QED Hilbert module and Interacting Fock spaces.

Publications of IIAS (Kyoto) (1997)

[AcLuVo95b] L. Accardi, Yun Gang Lu, Igor Volovich:

Nonlinear extensions of classical and quantum stochastic calculus and essentially infinite dimensional analysis.

in: Probability Towards 2000; Springer LN in Statistics 128 (1998) 1-33

[AcLuVo93] Accardi L., Lu Y.G., Volovich I.:

The Stochastic Sector of Quantum Field Theory.

Volterra Preprint N.138 (1993) Matematicheskie Zametki (1994)

[AcSchuvW88] Accardi L., Schurmann M., von Waldenfels W.: 
Quantum independent increment processes on superalgebras,

Math. ZeitSchr. 198 (1988) 451-477

[AcFrLu87] Accardi L., Frigerio A., Lu Y.G.:

On the weak coupling limit problem

in : Quantum Probability and Applications IV Springer LNM N. 1396 (1987) 20-58

[Berez98] Yu.M. Berezansky:

Commutative Jacobi fields in Fock space,

J. Func. Anal. 138 (1998) 163-190

[Berez97] Yu.M. Berezansky:

On the direct and inverse spectral problem for Jacobi fields,

Algebra i Analys (in russian) 9 (6) (1997) english transl. St. Petersburg Math. J. 9 (6) (1997)

[BeKo94] Yu.M. Berezansky, Yu.G. Kondratiev:

Spectral Methods in infinite dimensional analysis,

Kluwer (1994)

[BeLiLy95] Yu.M. Berezansky, V.O. Livinsy,, E.W. Lytvynov:

A generalization of Gaussian white noise analysis,

Methods Func. Anal. and Topol. 1 (1995) 28-55

[Bou88] A. Boukas:

Quantum stochastic analysis: a non-Brownian case,

$\mathrm{PhD}$ thesis, Southern Illinois University (1988)

[Bou91] A. Boukas:

An example of a quantum exponential process,

Monatsh. Math., 112(3) (1991) 209-215

[BruRo91] F.T. Bruss, L.C. Rogers:

Pascal processes and their characterization,

Stochastic Process. Appl. 37 (1991) 331-338

[Chiha78] T.S. Chihara: 
An introduction to Orthogonal polynomials,

Gordon and Breach (1978)

[Fei87] P. Feinsilver:

Discrete analogues of the Heisenberg-Weyl algebra,

Mh. Math., 104 (1987) 89-108

[FeSch93] P. Feinsilver and R. Schott:

Algebraic Structures and Operator Calculus, Vol. I: Representations and Probability Theory,

Kluwer Academic Publishers, Dordrecht (1993)

[FrSch99] U. Franz and R. Schott:

Stochastic Processes and Operator Calculus on Quantum Groups,

Kluwer Academic Publishers, Dordrecht (1999)

[Grig01] B. Grigelionis:

Generalized $z$-distributions and related stochastic processes,

Lithuanian Math. J. 41 (2001) 303-319

[Grig00] B. Grigelionis:

On generalized $z$-diffusions,

Preprint Vilnius (2000)

[Grig99] B. Grigelionis:

Processes of Meixner type,

Lithuanian Math. J. 39 (1999) 33-41

[Hi92] Hida T.:

Selected papers,

World Scientific (2001)

[HiKuPoStr93] Hida T., Kuo H.-H., Potthoff J., Streit L., White Noise. An Infinite Dimensional Calculus,

Kluwer Academic Publishers (1993) 185-231

[Hi75] Hida T.:

Analysis of Brownian Functionals. 
Carleton Mathematical Lecture notes 13 (1975)

[KoStWe98] Y. Kondratiev, L. Streit, W. Westerkamp, J. Yan:

Generalized functions in infinite dimensional analysis,

Hiroshima Math. J. 28 (1998) 213-260

[KoKuOl00] Y. Kondratiev, T. Kuna, M.J.Oliveira:

Analytical aspects of Poissonian white noise analysis,

submitted to: Infin. Dimen. Anal. Quant. Prob. Rel. Top.

[KoLy00] Y. Kondratiev, E. Lytvynov:

Operators of gamma white noise calculus,

Infin. Dimen. Anal. Quant. Prob. Rel. Top. 3 (2000) 303-335

[KoSiSt97] Y. Kondratiev, J.L. Silva, L. Streit:

Generalized Appell Systems,

Methods Funct. Anal. Topology 3 (3) (1997) 28-61

[Kuo96] H.-H. Kuo:

White Noise Distribution Theory

CRC Press, 1996

[Lytv02b] Eugene Lytvynov: Orthogonal decompositions for Levy processes with an application to the gamma, Pascal and Meixner processes,

arXiv:math.PR/0204087 v5, 27 May (2002)

[Lytv02a] Eugene Lytvynov:

Polynomials of Meixner's type in infinite dimensions Jacobi fields and orthogonality measures,

arXiv:math.CA/0203026 v5, 27 May (2002)

[Lytv95] E. Lytvynov:

Multiple Wiener integrals and non-Gaussian white noises: a Jacobi field approach,

Methods Func. Anal. and Topol. 1 (1995) 61-85

[Meix34] Meixner J.: 
Orthogonale Polynomsysteme mit einen besonderen Gestalt der erzeugenden Funktion,

J.Lond. Math. Soc. 9 (1934) 6-13

[NuSch00] D. Nualart, W. Schoutens:

Chaotic and predictable representations for Lévy processes,

Stochastic Process. Appl. 90 (2000) 109-122

[Priv02] Nicolas Privault:

Splitting of Poisson noise and Levy processes on real Lie algebra

IDA-QP 1 (2002)

[Schou00] W. Schoutens:

Stochastic Processes and Orthogonal Polynomials,

Lecture Notes in Statist., Vol. 146, Springer-Verlag, New York (2000)

[SchTeu98] W. Schoutens, J.L. Teugels:

Lévy processes, polynomials and martingales,

Commun. Statisti.-Stochastic Models 14 (1998) 335-349

[Sch93] M. Schürmann.

White Noise on Bialgebras, Lecture Notes in Math. 1544,

Springer-Verlag, Berlin (1993)

[Śnia99] P. Śniady.

Quadratic bosonic and free white noises.

Commun. Math. Phys: 211(3) (2000) 615-628 Preprint (1999)

[TsVeYo01] N. Tsilevich, A. Vershik, M. Yor:

An infinite-dimensional analogue of the Lebesgue measure and distinguished properties of the gamma process,

J. Funct. Anal. 185 (2001) 274-296 